\title{
A Contemporary Warming/Restraining Device for Efficient Tail Vein Injections in a Murine Fungal Sepsis Model
}

\author{
Junko Yano ${ }^{1}$, Elizabeth A. Lilly ${ }^{2}$, Mairi C. Noverr ${ }^{2}$, Paul L. Fidel ${ }^{1}$ \\ ${ }^{1}$ Center for Oral and Craniofacial Biology, Louisiana State University Health-School of Dentistry ${ }^{2}$ Department of Microbiology and Immunology, Tulane \\ University School of Medicine
}

\section{Corresponding Author}

Paul L. Fidel

pfidel@Isuhsc.edu

\section{Citation}

Yano, J., Lilly, E.A., Noverr, M.C., Fidel, P.L. A Contemporary Warming/ Restraining Device for Efficient Tail Vein Injections in a Murine Fungal Sepsis Model. J. Vis. Exp. (165), e61961, doi:10.3791/61961 (2020).

\section{Date Published}

November 6, 2020

\section{DOI}

$10.3791 / 61961$

URL

jove.com/video/61961

\section{Abstract}

In rodent models, tail vein injections are important methods for intravenous administration of experimental agents. Tail vein injections typically involve warming of the animal to promote vasodilation, which aids in both the identification of the blood vessels and positioning of the needle into the vessel lumen while securely restraining the animal. Although tail vein injections are common procedures in many protocols and are not considered highly technical if performed correctly, accurate and consistent injections are crucial to obtain reproducible results and minimize variability. Conventional methods for inducing vasodilation prior to tail vein injections generally depend on the use of a heat source such as a heat lamp, electrical/rechargeable heat pads, or pre-heated water at $37^{\circ} \mathrm{C}$. Despite being readily accessible in a standard laboratory setting, these tools evidently suffer from poor/limited thermo-regulatory capacity. Similarly, although various forms of restraining devices are commercially available, they must be used carefully to avoid trauma to the animals. These limitations of the current methods create unnecessary variables in experiments or result in varying outcomes between experiments and/or laboratories.

In this article, we demonstrate an improved protocol using an innovative device that combines an independent, thermally regulated, warming device with an adjustable restraining unit into one system for efficient streamlined tail vein injection. The example we use is an intravenous model of fungal bloodstream infection that results in sepsis. The warming apparatus consists of a heat-reflective acrylic box installed with an adjustable automatic thermostat to maintain the internal temperature at a pre-set threshold. Likewise, the width and height of the cone restraining apparatus can be adjusted to safely accommodate various rodent sizes. With the advanced and versatile features of the device, the technique shown here could become a useful tool across a range of research areas involving rodent models that employ tail vein injections. 


\section{Introduction}

The use of animal models involving rodents has been a staple of biomedical research. Numerous inbred and outbred strains, as well as genetically modified lines, are available and routinely used in laboratories worldwide. Tail vein injection is one of the essential methods in rodent models requiring intravenous (i.v.) administration of experimental agents. Generally, i.v. injections have major advantages over other routes of administration such as high absorbance rates by bypassing local tissues and the digestive tract and high tolerance to solutions of a wide range of concentrations or non-physiological $\mathrm{pH}^{1,2,3,4}$. Among other viable i.v. routes (e.g., saphenous veins, retro-orbital venous sinus), tail veins are considered the safest and most readily accessible blood vessel in rodents ${ }^{2,3,5,6}$. Hence, tail vein injection has been widely employed in an array of rodent models including infectious disease models ${ }^{7,8,9}$, transplantation of biological materials $^{10,11}$, evaluation of preclinical therapeutics ${ }^{12,13}$, and toxicological analyses ${ }^{14,15}$.

Consistency and accuracy of dosing are a critical requirement in successful tail vein injections. Surprisingly, quantitative and qualitative evaluation of tail vein injections in the literature implicates frequent mis-injections ${ }^{16,17}$. A study reported that twelve out of thirty injections performed by trained injectors left more than $10 \%$ of injected doses within the tail ${ }^{18}$. In addition, the safety and comfort of the animal receiving tail vein injections should be a primary concern during the procedure. Improper restraint can lead to injuries and a range of stress-related pathologies (e.g., weight loss, impaired immune responses) that could introduce substantial variables in sample quality ${ }^{19,20}$. These errors can cause increased variability in data and poor reproducibility, thus negatively affecting study outcomes.

Induction of vascular dilation in the animal is often necessary when performing tail vein injections due to the small diameter of the vessel, estimated to be $300 \mu \mathrm{m}$ in mice ${ }^{21}$. Vasodilation enhances the visibility of tail veins and aids in achieving optimal needle-vein alignment within the venous lumen. A variety of methods have been reported by laboratories such as immersing the tail in warm water ${ }^{22}$, applying heat to the tail using a warm drape, lamp, or hair $\operatorname{dryer}^{23,24}$, or placing the animal in a warm environment using a heating pad, incubator, or box combined with one of these heat sources $^{25}$. The devices can be either self-made for specific purposes or available from commercial suppliers. However, many lack thermoregulatory capabilities and if any, the device temperature is poorly maintained and often subject to variations in room temperature. Similarly, the use of a restraining device is necessary for tail vein injections as the use of anesthesia is not recommended 26,27 . Several types of laboratory-specific or commercial restraining devices have been developed. Typically, the animal is placed in a disposable $50 \mathrm{ml}$ conical tube ${ }^{4}$, slotted plexiglass walls, a tunnel, or cone ${ }^{28}$, all of which allow ample exposure of the tail while restricting movements of the animal. However, most restrainers have size limitations due to the rigidity of the materials. Furthermore, modern high-complexity devices, despite the practical and sophisticated designs, do not appear to be feasible for injections involving large groups of animals $^{22}$.

Mouse models of bloodstream infection and associated sepsis are a prime example of situations requiring the 
use of this technique. Among all microbial etiology of severe clinical sepsis, fungal sepsis is often a fatal condition with mortality rates of $>40 \%$ despite antifungal therapy $^{29}$. In fact, infection by Candida albicans has been reported as the fourth leading cause of hospital-acquired bloodstream infection (candidemia) 30,31 . In intra-abdominal candidiasis, microorganisms in the gastrointestinal tract can disseminate via the bloodstream and cause polymicrobial sepsis with an even greater mortality $32,33,34$. As most nosocomial candidemia cases emerge from contaminated central line catheters or indwelling medical devices 35,36 , i.v. inoculation with $C$. albicans by tail vein injection can closely mirror human sepsis development and has been a staple method in a mouse model of hematogenously disseminated candidiasis $^{37,38}$. In this model, mortality that occurs in days can be extended or shortened by adjusting the $C$. albicans i.v. inoculum $39,40,41$.

Recently, our laboratory has developed an innovative protocol for an optimally streamlined tail vein injection using an innovative device equipped with a thermoregulated warming unit, paired with an adjustable restraining unit, in one convenient system. This protocol allows researchers to perform tail vein injections in an accurate and timely manner, while animals can be safely conditioned and restrained for the procedure with minimal distress. The techniques demonstrated here, with the use of the advanced warming and restraining device, could serve as a useful tool in various research areas employing rodent models.

\section{Protocol}

All animal protocols involving tail vein injections and use of the warming/restraining device were reviewed and approved by the local Institutional Animal Care Committee (IACUC).

\section{Preparation}

1. Acclimate animals in the housing environment for at least 1 week, and allow food and water ad libitum.

NOTE: For most new users of this injection technique, animal strains with white or light-colored fur may be preferable as the tail veins are readily visible through the skin. Dark-colored strains of mice (e.g., C57BL/6) or rats (e.g., Brown Norway) have deeply pigmented tails, resulting in a weak color contrast against the vein. It is highly recommended that new users receive adequate training until proficiency is attained

2. Agents for tail vein injection

1. Prepare all test agents and solutions aseptically. When administering organisms or cellular materials, take precautions during all steps of processing to maintain pyrogen-free conditions.

2. Use only normal saline $(0.9 \% \mathrm{w} / \mathrm{v}$ sodium chloride $)$ or balanced salt solutions such as phosphate-buffered saline (PBS) as vehicles for tail vein injection.

CAUTION: Never use water, oil, or viscous solutions owing to the potential risk of vascular damage. A wide range of $\mathrm{pH}(4.5-8.0)$ is tolerable because of the buffering effect of blood and fast blood flow rates in rodents. However, highly acidic or alkaline solutions can result in unnecessary tissue damage at the injection site and should be avoided.

3. Limit the volume and frequency of injection to a minimum. Use the recommended volumes for mice and rats ( $\leq 200 \mu \mathrm{L}$ and $\leq 500 \mu \mathrm{L}$, respectively) at body temperature before injection to minimize stress to the animal $^{3}$. 
4. Ensure that each preparation of the syringe and needle is free from air bubbles in the solution; if bubbles are present, purge them completely to prevent the risk for embolism.

NOTE: Typically, $1 \mathrm{~mL}$ syringes with $27 \mathrm{G}, 1 / 2$-in needles are adequate for most tail vein injections.

5. Use appropriate personal protective equipment (PPE) required by local IACUC with the minimum of disposable or dedicated gowns and latex or nitrile gloves. Use of safety glasses is highly recommended when performing tail vein injection.

3. The warming and restraint device

1. Carefully inspect all components before use to ensure that the device is free of any defects (Figure 1).

2. Warming device initialization (Figure 2A)

1. Place the warming unit on a clean flat benchtop, and power the device on. Ensure that the thermostat power indicator lamp is lit green. Place bedding materials inside the warming chamber to keep the area dry and retain heat.

\section{Restraint device setup (Figure 2B)}

1. Place the restraint unit alongside the warming unit, and determine the appropriate cone sizes for the animal. If necessary, manually adjust the base widths of the pliable aluminum cone to provide adequate restraint for the animal. Alternatively, replace the cone with custom-fitted models to accommodate mice or rats of varying body sizes.

\section{Tail vein injection}

1. Apparatus adjustments

1. Setting the internal temperature
1. Using the control dial, set the thermostat at the desired temperature. Ensure that the heater indicator is lit red, and that the light bulb illuminates. Monitor the internal temperature display carefully while the bulb is illuminated (heating). The thermostat inactivates the bulb automatically once a target temperature has reached, approximately in 10-15 min.

NOTE: Setting a temperature higher than the ambient temperature activates the heater. In general, the recommended housing temperature in standard vivarium conditions vary, ranging from 20 to $26{ }^{\circ} \mathrm{C}$, while the neutral (i.e., comfortable) temperature for laboratory mice is considered to be between 30 and $32{ }^{\circ} \mathrm{C}^{42}$. Therefore, it is recommended that the internal temperature of the warming chamber be elevated slightly higher than thermoneutrality, approximately at $32-36$ ${ }^{\circ} \mathrm{C}$. Never set the thermostat above the body temperature.

\section{Positioning the restraint platform}

1. Using the height adjustment knob, adjust the cone height to the optimal level for the user.

2. Heat treatment (Figure 3A)

1. Once the target temperature has been reached (32$\left.36{ }^{\circ} \mathrm{C}\right)$, gently transfer the animals from the housing cage into the warming chamber.

NOTE: Heat treatment for $5-10 \mathrm{~min}$ is sufficient to induce vasodilation and enhance the visibility of tail veins. However, animals can be safely held in the thermoregulated chamber for the duration of the procedure (typically $20-30$ min with no sign 
of hyperthermia). The warming chamber can safely contain 4-6 mice or one rat.

2. Monitor the animal for any signs of acute heat stress (e.g., rapid respiration, lethargy, jumping escape behavior).

CAUTION: Animals exhibiting signs of hyperthermia should be returned to their cage and monitored until they resume normal activity prior to reuse. If this is because of the internal temperature exceeding the optimal range, ensure that the warming device is turned off.

3. Injection steps

1. Lift the animal by the base of the tail, and remove it from the warming chamber. Introduce the animal onto the cone opening of the restraining unit.

CAUTION: Never lift mice from the tail end; this can result in serious injuries. Alternative methods of handling should be used for obese or pregnant mice $^{28}$.

2. As the animal grasps on the far edge of the cone with its forelegs, gently pull the tail backward and pass the tail through the open slit. Secure the hind end of the animal at the base of the cone with one hind leg protruding out from the cone so the lateral vein is shown at a position of 12 o'clock. Either hind leg can be protruded as there are two lateral veins, one on each side (Figure 3B).

3. Grasp the tail at the mid- to two-thirds-length with the non-dominant hand between the thumb and forefinger, putting slight tension on the lateral vein to maintain the tail positioning and vasodilation.
NOTE: Enhanced visibility of the dilated veins by heat treatment enables the user to quickly determine an injection site for the best results (Figure 4).

4. Wipe the skin of the injection site with a gauze sponge or pad moistened with $70 \%$ alcohol. Clean as gently and quickly as possible to avoid irritation to the tail. NOTE: This procedure can be omitted at the discretion of the institutional IACUC.

5. Hold the syringe with the dominant hand, and position the needle parallel to the tail. Insert the needle toward the direction of the blood flow, bevel up at a $10-15^{\circ}$ angle (Figure 5A-B), and advance further into the lumen of the vein by penetrating $2-4 \mathrm{~mm}$ (Figure $5 \mathrm{C}$ D). Slowly inject the solution.

NOTE: If the injection is successful, no resistance on the plunger should be felt, and the fluid can be seen moving through the vein. In case of resistance or white blisters above the injection site, remove the needle and attempt a second injection at a site above the original needle placement. Do not attempt to inject below the initial injection site as the fluid will release through the initial site. If injection of one lateral vein is unsuccessful, reposition the animal to the opposite side and make more attempts on the contralateral vein. The maximum number of attempts will depend on where one starts the attempted injection along the vein and the swelling that may occur with missed attempts. Consult the institutional IACUC regulations for mis-injections and associated injuries.

6. Remove the needle, and press firmly with the thumb to prevent backflow of the injected solution and/or blood. Continue to apply gentle compression with a clean gauze/wipe or tissue until bleeding has stopped (Figure 6). 
7. Return the animal to its cage, and monitor for at least $5 \mathrm{~min}$. Ensure that the animal resumes normal activity without further bleeding.

\section{A murine model of fungal bloodstream infection and sepsis}

1. Mouse strains

1. Acclimate female Swiss Webster outbred mice at 6 weeks of age per institutionally recommended guidelines. Alternatively, use inbred/genetically modified strains (e.g., C57BL/6 background) for this protocol with modified inocula (see NOTE).

NOTE (see Discussion for detail): Tail veins of mice with dark fur are often less visible than those with lighter fur due to the deeply pigmented tail (Figure 4). There is varying susceptibility to fungal sepsis/lethality among different mouse strains. Use of mouse strains other than Swiss Webster may require additional protocol optimization by considering relevant factors (e.g., genetic background, age, sex, body size) that could influence host immune status. For example, a lethal challenge in C57BL/6 mice typically requires higher inocula (up to $10 x$ ) to achieve the level of mortality seen in Swiss Webster mice.

2. Microorganisms

1. For a lethal challenge (sepsis), streak frozen stocks of Candida albicans strain DAY185 (or strains of choice) onto Sabouraud dextrose agar and incubate at $30^{\circ} \mathrm{C}$ for 2 days.

2. Transfer a single colony into $10 \mathrm{~mL}$ yeast extractpeptone-dextrose broth, and culture to the stationary phase of growth for $18 \mathrm{~h}$ at $30^{\circ} \mathrm{C}$ with shaking.

3. Inoculum solutions
1. On the day of a lethal challenge, collect the broth culture, and wash the pellet 3 times by centrifugation $(800 \times g)$ in sterile PBS.

2. Identify viable yeast cells by trypan blue dye exclusion, and enumerate using a hemocytometer. Adjust the cell concentration to $1 \times 10^{6}$ cells $/ \mathrm{mL}$ in sterile PBS at room temperature.

NOTE: Each animal will receive $100 \mu \mathrm{L}$ of the inoculum solution. Prepare an excess volume of the inoculum $(>500 \mu \mathrm{L})$ to allow for potential loss during the injection procedure. The final inoculum is $1 \times 10^{5}$ cells per mouse. The inoculum volume can be increased up to $200 \mu \mathrm{L}$ by adjusting the cell concentration accordingly.

CAUTION: The fungal inoculum solution must be kept at room temperature prior to injection. Warming the inoculum solution to body temperature may induce a morphological change from yeast cells to hyphae. Contrarily, bolus i.v administration of cold solutions can rapidly lower body temperature of the animal and should be avoided.

4. Intravenous inoculation

1. Warm the animals, and induce vasodilation by following the procedures in section 2 .

2. Inject $100 \mu \mathrm{L}$ of the inoculum solution into the tail vein using a $1 \mathrm{~mL}$ syringe with a $27 \mathrm{G}, 1 / 2$-in needle.

\section{Post-inoculation monitoring}

1. Monitor the animals for the following signs of sepsisinduced morbidity: i) fur aspect (e.g., smooth, ruffled), ii) activity (e.g., moving freely, nonresponsive), iii) posture (e.g., hunched, stiff), iv) behavior (e.g., slow, no relocation), v) chest movements (e.g., 
normal breathing, dyspnea), vi) eyelids (e.g., open, (closed $)^{43}$.

6. Sepsis scoring

1. Score the observed morbidity according to a modified Mouse Clinical Assessment Score for Sepsis (MCASS) in a four-point grading scale from 0 to 3 in each category: 0 , normal; 1 , mild; 2 , moderate; 3 , severe ${ }^{43}$.

7. Optional protocol: Vaccination against fungal sepsis

1. Fourteen days prior to a lethal challenge, inoculate mice with Candida dubliniensis strain Wü284 or attenuated C. albicans strains, such as $\Delta$ efg $1 / \Delta c p h 1$ mutant $\left(1 \times 10^{5}\right.$ cells per mouse), as described in sections 3.2-3.4 in lieu of C. albicans DAY185.

2. Conduct a lethal challenge in the vaccinated mice, as described in sections 3.2-3.4, and monitor for the signs of sepsis-induced morbidity described in sections 3.5-3.6.

\section{Representative Results}

The temperature inside the warming chamber is continuously detected by the internal sensor and auto-regulated by the thermostat. First, the control dial of the thermostat was positioned at $78,85,90$, or $95^{\circ} \mathrm{F}\left(26,29,32\right.$, or $\left.95^{\circ} \mathrm{C}\right)$ to select set temperatures. Once the heater was activated (Figure 7, yellow dots), heat emission by the light bulb rapidly raised the internal temperature during the first 5-15 min, depending on the set temperature. The heater inactivated the light bulb if the detected internal temperature exceeded the set temperature (gray dots). The initial peak temperatures should rise to 5$7{ }^{\circ} \mathrm{C}$ above the set temperatures in all groups to offset the temperature loss during animal transfer. Subsequently, the device continues to repeat the heat cycle automatically and maintains the warming chamber at the set temperature.

An example of experimental data obtained by successful tail vein injections using the current protocol is shown in Figure 8. In a mouse model of bloodstream candidiasis resulting in sepsis, an i.v. challenge with Candida albicans $\left(1 \times 10^{5}\right.$ cells per mouse) in Swiss Webster mice caused a rapid onset of sepsis and dissemination of the organisms, leading to high mortality within 3-4 days (open dots) (Figure 8A). In contrast, animals could be protected from sepsis by prior i.v. pre-immunization/vaccination with an avirulent yeast strain, Candida dubliniensis, achieving $>95 \%$ survival following the lethal i.v. challenge with virulent $C$. albicans (solid dots). These results in progressive mortality vs. vaccine-mediated protection were obtained reproducibly in four independent experiments (Supplemental Figure 1). Similar protection could be achieved using other avirulent yeast strains such as attenuated $C$. albicans mutants $(\Delta e f g 1 / \Delta c p h 1)$ (data not shown). Sepsis could be monitored as well and correlated to mortality; the unvaccinated animals with lethal infection had a significant increase in sepsis-induced morbidity, whereas the vaccinated group exhibited minimal symptoms following the lethal challenge (Figure 8B). 

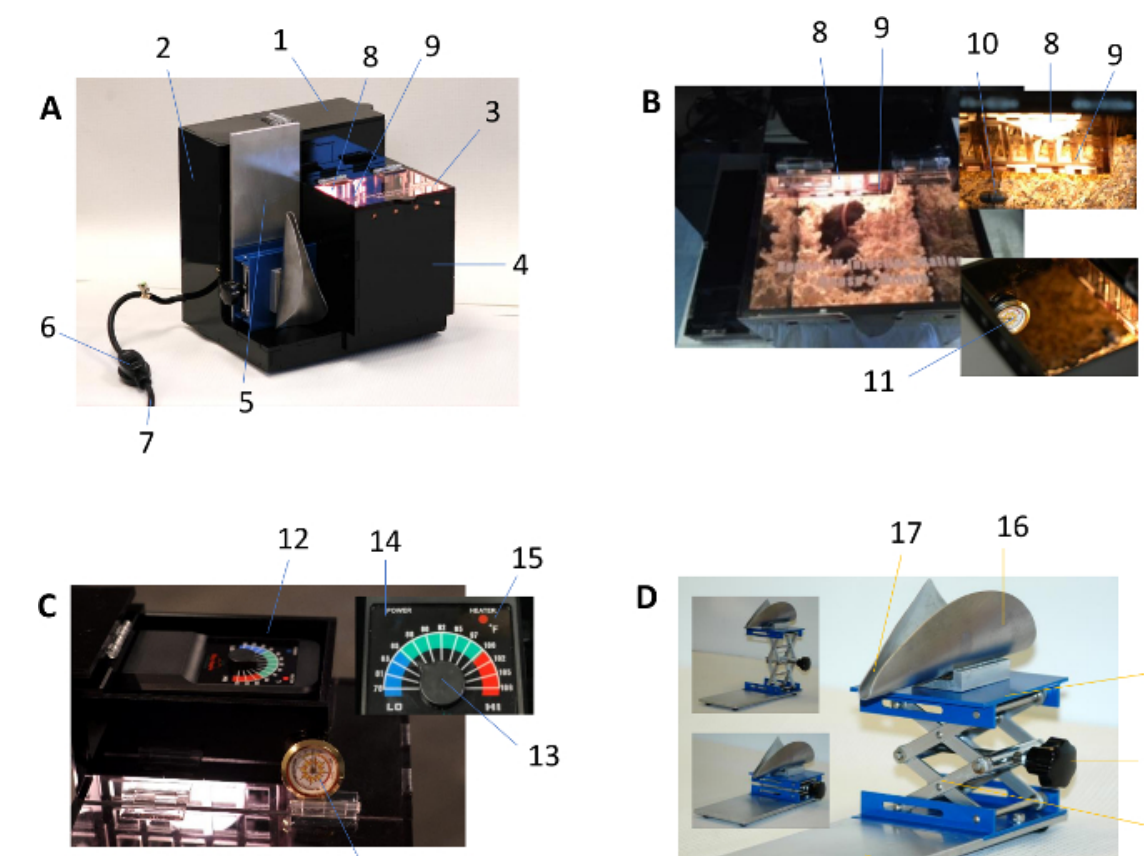

11

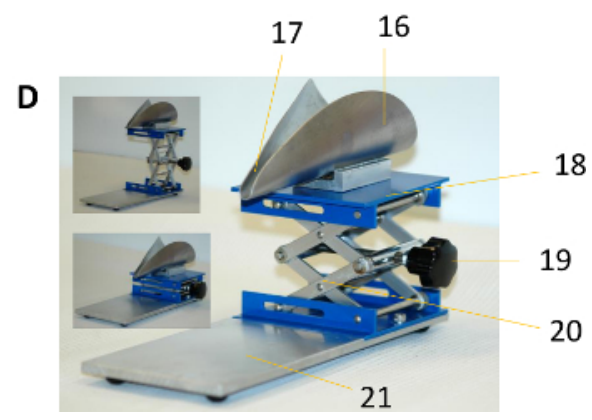

Figure 1: Description of the rodent warming and restraining device. $(\mathrm{A})$ shows the exterior view of the warming device, which consists of:

1. Thermostat cover - lift upward by the handle to expose the thermostat

2. Electrical enclosure - sealed permanently for protection

3. Chamber lid - lift upward during animal transfer to/from

4. Warming chamber - removable, cover the floor with bedding before use

5. Restraint apparatus - stowable with the heating device while not in use

6. Power switch - inline rocker switch for main on/off functions

7. Power cord - voltage/current: $120 \mathrm{~V} / 10 \mathrm{~A}$

(B) shows the interior of the warming device:

1. Incandescent light bulb - light output at 100 Watts
2. Light bulb protective shield - removable for bulb replacement

3. Temperature sensor probe - located inside the chamber

4. Internal temperature thermometer - place inside the chamber for monitoring temperature

(C) shows components of the warming device thermostat:

1. Internal temperature thermometer

2. Thermostat - auto-regulates the heater

3. Setpoint control lever - minimum/maximum: $78^{\circ} \mathrm{F} / 108^{\circ} \mathrm{F}$ $\left(25^{\circ} \mathrm{C} / 42^{\circ} \mathrm{C}\right)$

4. Thermostat power indicator - green light indicates normal operation

5. Thermostat heater indicator - lit red during heating cycle 
(D) shows components of the restraining device:

1. Cone - pliable aluminum sheet designed for rodent restraint

2. Tail channel - shaped to permit smooth positioning of the tail

3. Cone lift platform - provides sturdy lift of the cone base
4. Height adjustment knob - designed for manual height adjustment

5. Scissor jack - height range from 45-140 mm (1.77-5.52")

6. Support plate - installed with rubber feet to provide stability Please click here to view a larger version of this figure.
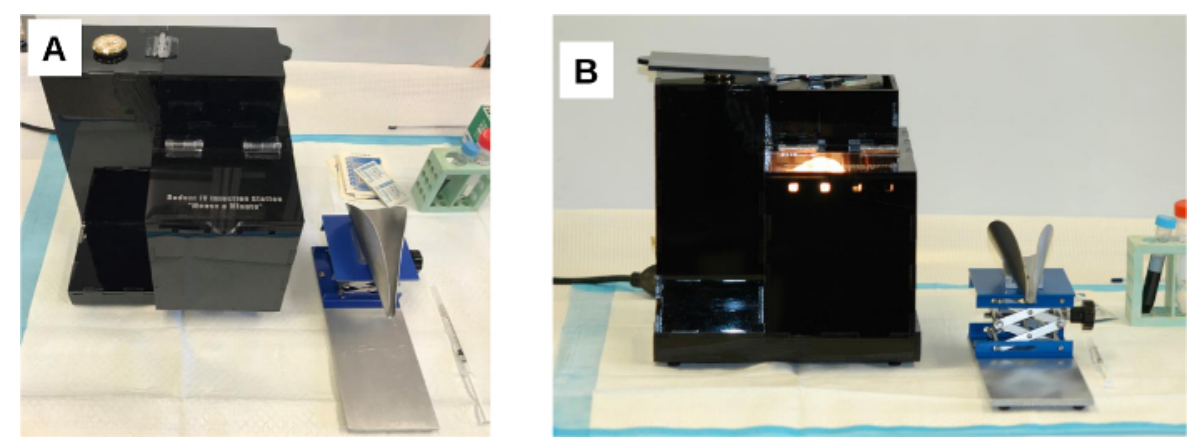

Figure 2: The rodent warming and restraining device. (A) Prior to use, the two parts of the device are placed side by side on a clean bench top. (B) Once the warming device is powered on, the thermostat activates the heater. The light bulb remains lit and emits heat until the warming chamber has reached the set temperature. The warming device automatically repeats the heat cycle to maintain the internal temperature. Please click here to view a larger version of this figure. 

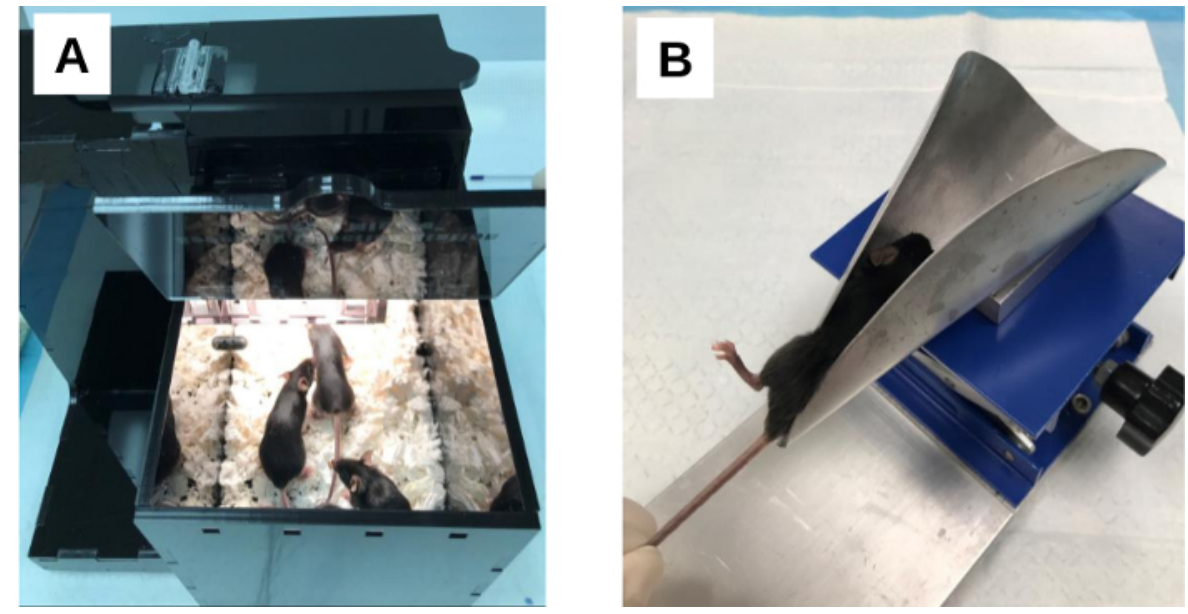

Figure 3: Mice (C57BL/6) placed in the warming and restraining device. (A) Mice receiving heat treatment for vasodilation. The animals (4-6 mice per treatment) are transferred from their housing cage into the warming chamber of the device and heat-treated for a minimum of 5-10 min. (B) A mouse restrained for tail vein injection. The mouse is transferred from the warming chamber into the cone opening of the restraining device with its tail passing through the open slit. The mouse is gently pulled backward to the far edge of the cone until the base of the tail reaches the tip of the cone. As the animal is drawn toward the base of the cone with a gentle lateral rotation, one hind leg is positioned upward so that it protrudes out from the open slit allowing the lateral tail vein to be positioned at 12 o'clock. Please click here to view a larger version of this figure. 

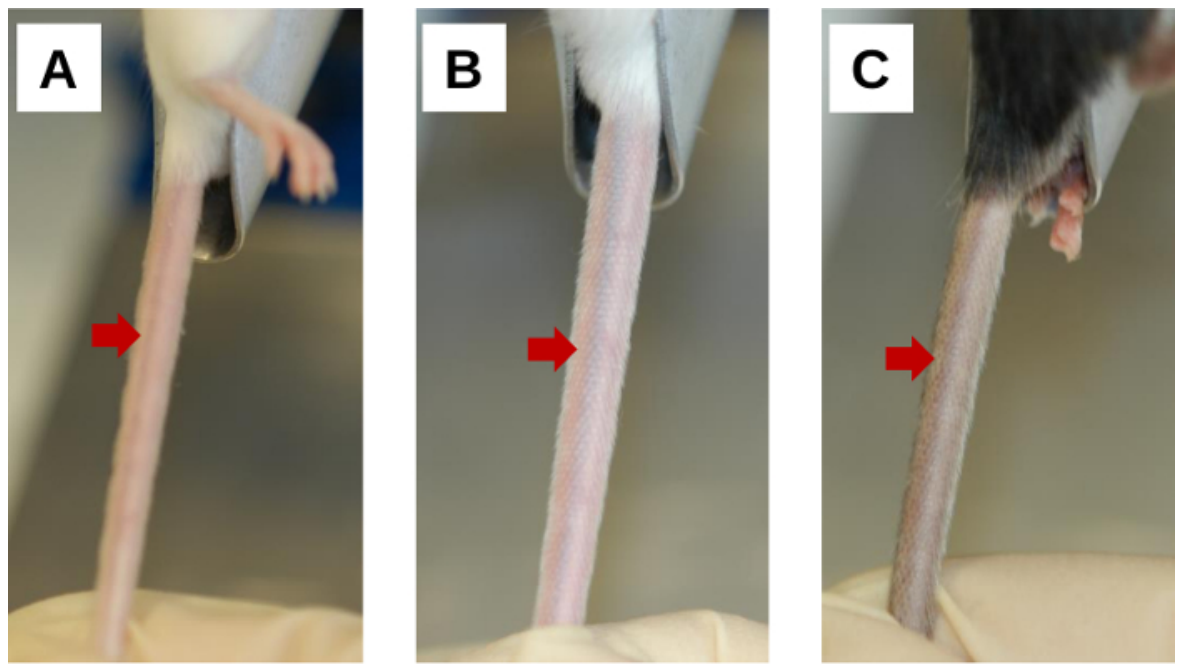

Figure 4: Identification of the lateral tail veins in mice. (A) The tail of an untreated Swiss Webster mouse. The mouse is placed in the restraining device without prior heat treatment for vasodilation. The lateral tail vein can be identified as a thin dark vessel that courses under the skin. (B) The tail of a Swiss Webster mouse treated with the warming device for 10 min. The heat-treated mouse is restrained for tail vein injection. The lateral tail vein is readily visible through the skin due to the enlarged vessel diameter induced by vasodilation. (C) The tail of a C57BL/6 mouse treated with the warming device for 10 min and restrained for tail vein injection. Vasodilation enhances visibility of the tail vein through the deeply pigmented skin although the vein is not as readily visible as in the light-colored Swiss Webster mice due to a weak color contrast against the vein. Red arrows denote the location of the lateral tail vein. Please click here to view a larger version of this figure. 

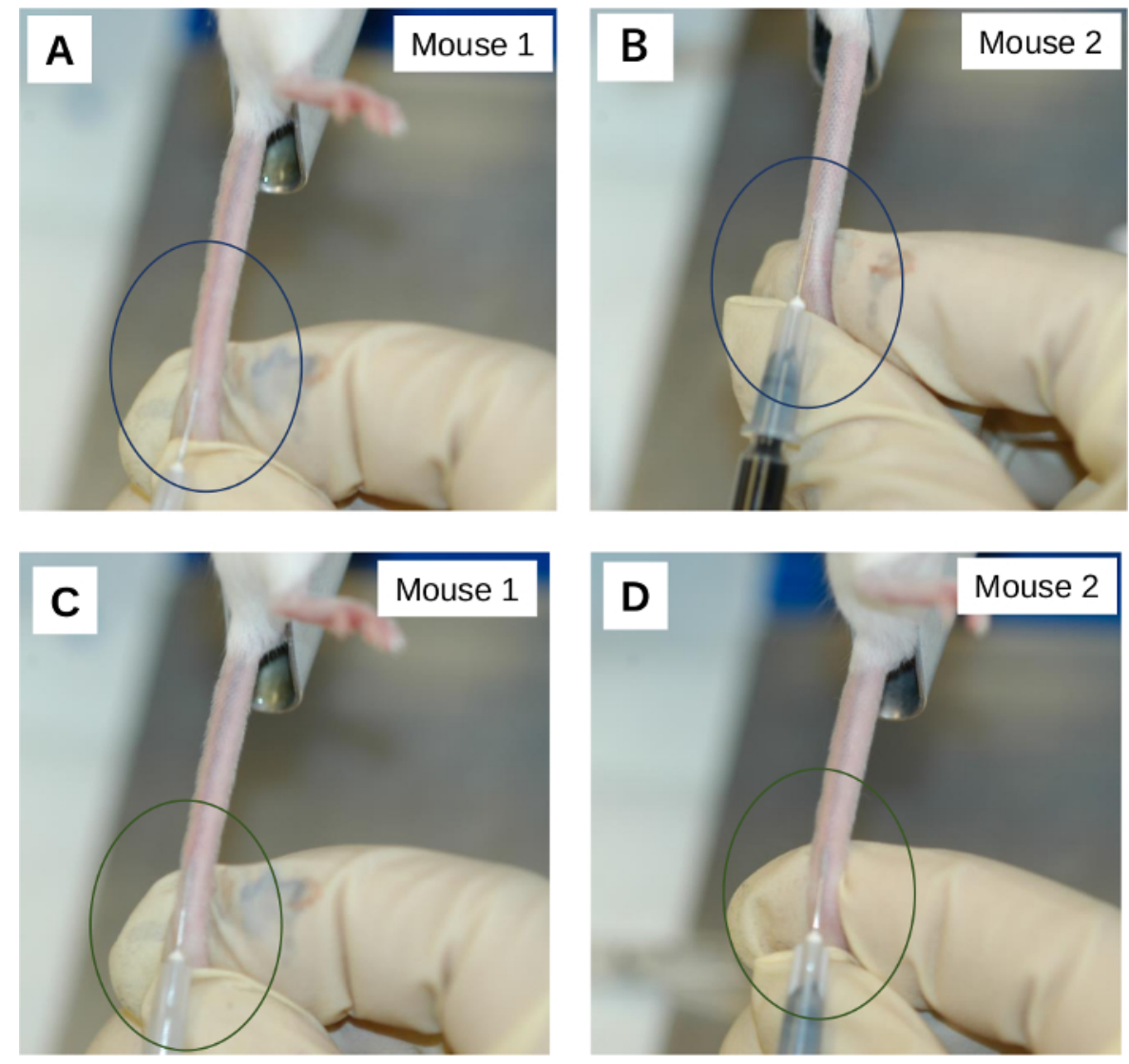

Figure 5: Tail vein injection performed in heat-treated mice (Swiss Webster). (A-B) Needle insertion into the lateral tail vein at the injection site. The needle $(27 \mathrm{G}, 1 / 2$-in) is positioned parallel to the tail vein with the bevel up and pointed toward the blood flow and inserted. (C-D) Needle placement in the tail vein and injection. The tip of the needle is further advanced 2-4 $\mathrm{mm}$ into the lumen of the vein. The thumb is positioned on the plunger of the syringe, and the desired volume is dispensed with slow and steady pressure. Oval circles indicate injection sites. Please click here to view a larger version of this figure. 

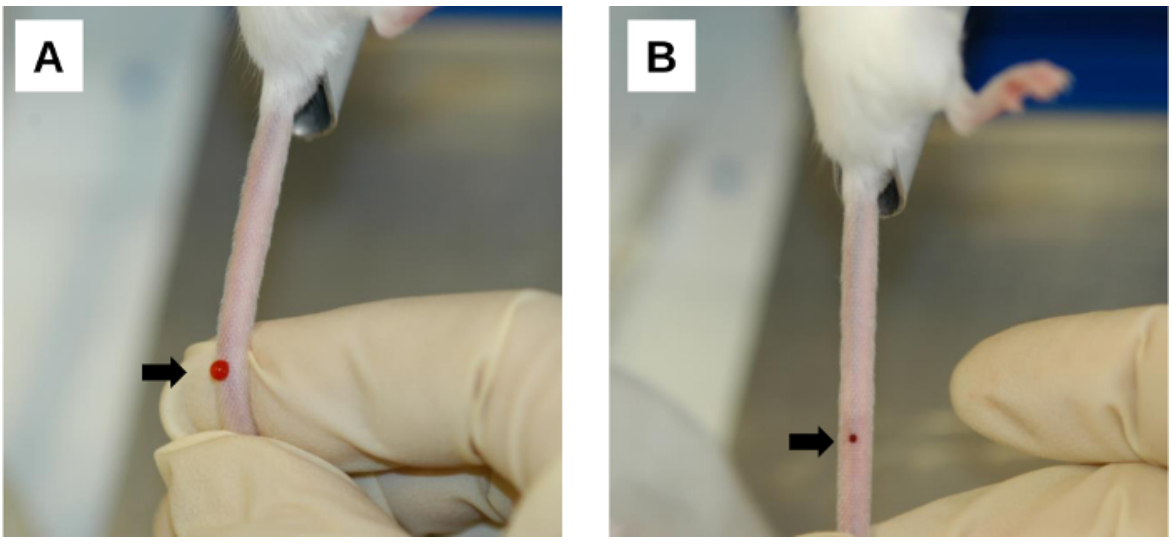

Figure 6: Post-injection procedure. (A) A bleeding area at the injection site. Bleeding and backflow of the injected solution occur immediately after needle removal. This can be minimized by applying firm compression on the injection site with the thumb. (B) Blood clot formation at the injection site. Gentle compression with a clean gauze/wipe facilitates blood clotting on the injection wound. Arrows denote injection sites. Please click here to view a larger version of this figure.

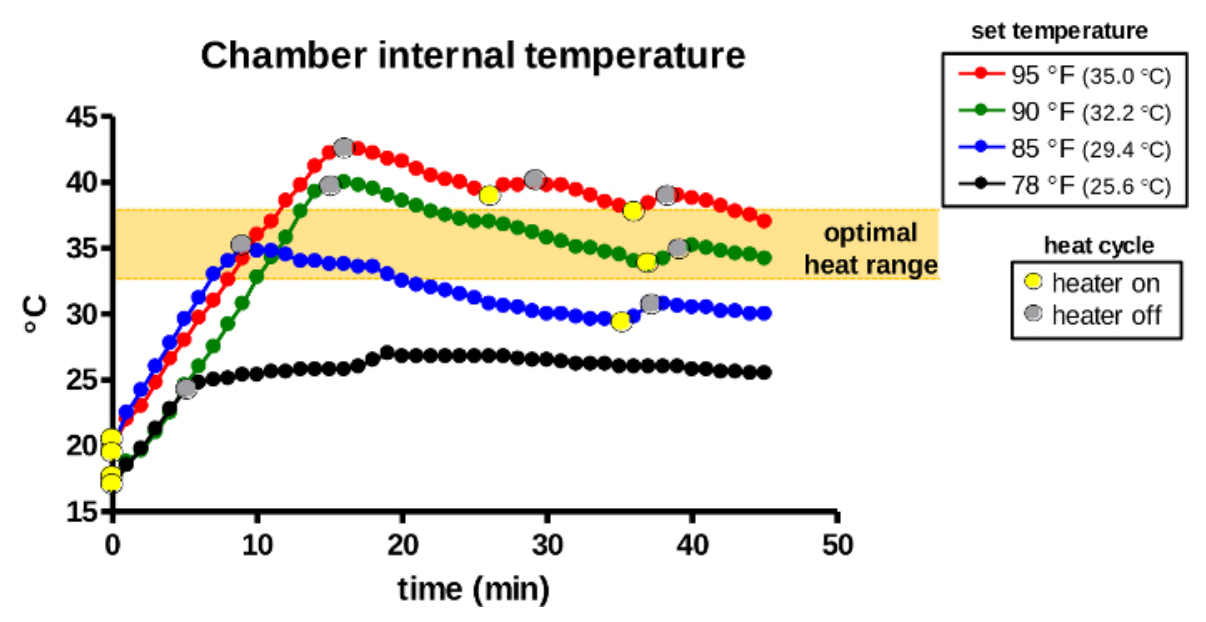

Figure 7: The internal temperature of the warming chamber during use. The warming device was activated for warming at the designated set temperatures. The warming chamber of the device was monitored for the internal air temperature and heat cycles (light bulb on/yellow dots, off/gray dots) were recorded over $45 \mathrm{~min}$. The orange area indicates the optimal temperature range for induction of vasodilation in rodents. Please click here to view a larger version of this figure. 
A.

Survival

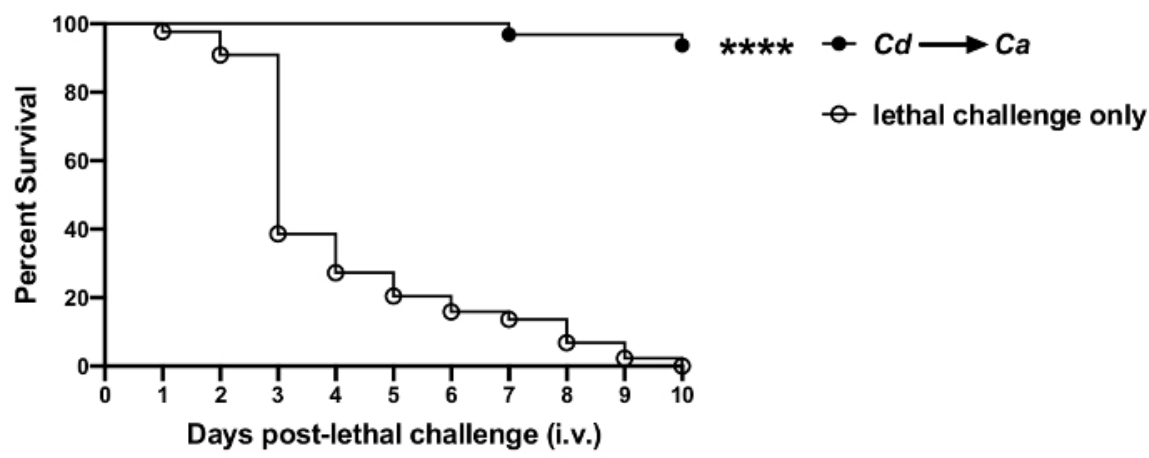

B.

Sepsis score

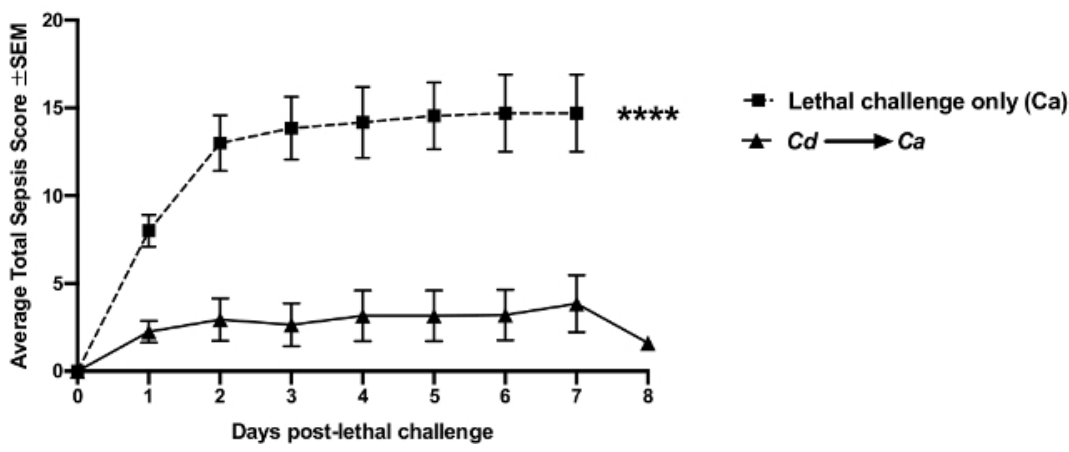

Figure 8: Sepsis mortality vs. vaccine-mediated protection following a lethal challenge with Candida albicans. Mice (8 week-old Swiss Webster females) were vaccinated intravenously with avirulent live Candida dubliniensis Wü284 (Cd), followed by a lethal intravenous challenge with wild-type $C$. albicans DAY 185 ( 1 × $10^{5}$ cells per mouse) 14 days later. (A) Mortality was assessed over 10 days following the lethal challenge. (B) Animals were monitored for sepsis morbidity and scored according to a modified Mouse Clinical Assessment Score for Sepsis (M-CASS) ${ }^{43}$. Data are cumulative of 4 independent experiments with 10 mice per group and analyzed using the Mantel-Cox log-rank test. ${ }^{* * * *} p<0.0001$. SEM, standard error of the mean. Please click here to view a larger version of this figure.

\section{Supplemental Figure 1: Reproducibility of sepsis} mortality and vaccine-mediated survival from a bloodstream Candida albicans challenge. Each panel represents data from four independent experiments included in a cumulative result shown in Figure 8A. Each experiment was conducted using 10 mice per group and analyzed using the Mantel-Cox log-rank test. Ca, Candida albicans. Cd,
Candida dubliniensis. ${ }^{* * * *} p<0.0001 .{ }^{* * *} p<0.01$. Please click here to download this figure.

\section{Discussion}

Consistent and accurate dosing are key requirements for experimental reliability in animal models. This is especially important in cases of i.v. administration where systemic bioavailability of injected agents is considerably higher/faster than with other administration routes ${ }^{3}$. Thus, errors in tail vein 
injection could have a detrimental impact on study outcomes. Historically, intraperitoneal (i.p.) injection, rather than i.v., has been the most common method for systemic access in rodents due to technical simplicity and convenience. However, administration routes become more crucial when translating preclinical readouts from animals into clinical settings. Hence, there is a need for continuous improvement in rodent protocols that could facilitate successful tail vein injection.

The key advancement in the present protocol is the innovative thermoregulated warming device that enables effective induction of vasodilation in rodents, which dramatically improves the visibility of tail veins and needle alignment. Heating methods that are poorly thermoregulated (e.g., lamps), topical vasodilators or skin irritants (e.g., xylenes) are not only unreliable, but are also unsafe for the animal and should be avoided ${ }^{44}$. Contrary to other conventional methods, such as immersing the tail in warm water, the autoregulation capability of this device can safely condition multiple animals simultaneously. In addition, this protocol is strengthened further by using the optimally designed restraining device and allowing fast and secure immobilization of the animal in a position that best displays the lateral tail vein.

The transparent tubal formats seen in many current restrainers, though practically well-designed, require more handling time with each animal, thus prolonging the restraining process ${ }^{45}$. This can be more problematic in rodent strains with aggressive traits that offer limited cooperation 46,47 . In contrast, the semi-enclosed cone structure of the restraining device permits quick positioning of the animal and aids in minimizing the duration of restraint. Together, the streamlined protocol using the innovative, highly optimized warming/restraining system accelerates the injection procedure, allowing for quick and effective dosing of large groups of animals. In our laboratory, we typically complete an entire injection procedure of 30 mice from heat treatment to post-injection monitoring within $1 \mathrm{~h}$ using this protocol.

Despite the advanced features, this device has some apparent disadvantages: the first is the cost of the device and routine light bulb replacement in the warming chamber. However, in addition to the efficiency and speed of injections, the device is durable for repeated use and compatible with most common disinfectants, permitting thorough cleaning of the device between uses. Together, this offsets the initial investment. Second, in situations with limited workspace, a drawback to this protocol may be the requirement for a dedicated bench area large enough to place the two units, side by side, while performing the injection. However, because the device can be utilized broadly across several rodent protocols involving i.v. injections, it is possible that the device could serve as a core instrument similar to other communal vivarium equipment such as isoflurane vaporizers. Regardless, the two units are easily portable and can be bundled and stowed while not in use.

The i.v. lethal challenge model of murine fungal sepsis described in this protocol closely mimics C. albicans bloodstream infections in humans and has been extensively used to study fungal virulence, test efficacy of antifungal therapies, and characterize host immune responses to infection $^{37,39,48}$. To achieve a reproducible infection, i.v. inoculation via tail vein injection is the most vital step of the protocol to ensure accurate delivery of the organisms into the bloodstream. In fact, animals respond very differently to varying levels of Candida i.v. challenges; administration of too 
low amounts of inoculum will result in unwanted spontaneous recoveries, whereas animals receiving too high doses will succumb prematurely. The specific window of inoculum sizes for a given organism to induce a consistent level of sepsis/ mortality largely depends on both fungal strains and mouse strains.

The current protocol using Swiss Webster mice at the inoculum of $1 \times 10^{5}$ wild-type $C$. albicans reproducibly induced the onset of sepsis morbidity within 1 day, followed by progressive mortality resulting in $100 \%$ lethality by $5-$ 7 days. In contrast, inocula higher than $1 \times 10^{5}$ typically lead to accelerated deaths (i.e., $1-2$ days at $1 \times 10^{6}, 3-4$ days at $5 \times 10^{5}$ ), and those lower than $1 \times 10^{5}$ are sublethal. In line with numerous reports in the literature, the use of non-albicans Candida species in lieu of $C$. albicans results in significantly diminished lethality ${ }^{40,49}$. Additionally, the choice of mouse strains, or even the origin of colonies, can have a considerable impact on infection outcomes due to varying susceptibilities between mouse strains, as reported by others $39,40,41,50,51,52,53,54,55$. Hence, both should be taken into consideration when designing experiments.

Following a lethal i.v. challenge, fungal cells spread rapidly through the bloodstream and begin to invade multiple organs, among which the most affected are the kidneys ${ }^{41}$. Other organs affected are the brain, spleen, and bone marrow ${ }^{48,56}$. Regardless, acute sepsis is the ultimate cause of death at the early time points ${ }^{37}$. As shown in the representative results, sepsis severity can be quantitatively assessed by the Mouse Clinical Assessment Score for Sepsis (M-CASS) based on exhibited signs of a sepsis condition in challenged animals $^{43,57}$. Among the several surrogate markers of lethal sepsis, hypothermia has been suggested as a critical predictor for imminent death in both clinical and experimental sepsis $^{43,58,59}$.

Although no formal studies have been conducted to directly compare inbred vs. outbred mice in this model, data obtained from the current protocol using outbred Swiss Webster mice are exceptionally reproducible in various sepsis parameters, despite the presumed genetic heterogeneity. Generally, a pattern of mortality that falls within $3-5$ days is a firm model of acute sepsis, as evidenced by rapid elevation in sepsis morbidity and levels of inflammatory markers within hours of post-lethal challenge $\mathrm{e}^{50,51}$. For longer survival times (7-10 days), mortality is likely the result of microbial burden leading to lethal tissue damage in target organs and the central nervous system. The choice of sepsis or microbial burden can be applied as necessary for evaluating immune functions or responses to anti-inflammatory regimens or antifungal therapies/vaccines, as determined by the inoculum used.

In addition to the i.v. lethal challenge model, intra-abdominal infection with $C$. albicans in mice via an i.p. challenge can also lead to disseminated candidiasis and subsequent sepsis, although co-inoculation with the bacterial pathogen, Staphylococcus aureus, synergistically enhances mortality compared to $C$. albicans mono-infection $51,60,61$. In the i.p. lethal challenge model, substantially higher microbial inocula $\left(1.75 \times 10^{7}\right.$ C. albicans $/ 8 \times 10^{7} \mathrm{~S}$. aureus per mouse) are required to cause polymicrobial peritonitis and dissemination of the organisms from the abdominal cavity into the bloodstream. Similarly, gastrointestinal infection with C. albicans in mice treated with immunosuppressive and/ or mucosal-damaging agents leads to translocation of the fungal cells into the bloodstream and results in fungal sepsis $^{62,63}$. Despite the distinctive inoculation routes, the mechanism of ensuing fungal sepsis is largely analogous 
between the three disease models, involving an uncontrolled systemic proinflammatory response to Candida that leads to organ failure $37,51,61$. Similarly, in humans, it is this process of the host response, not simply candidemia, that causes the high morbidity/mortality associated with hematogenously disseminated candidiasis acquired in health care settings 64,65 .

Using the current fungal sepsis model, we demonstrate here that protection against lethal $C$. albicans infection can be achieved by i.v. pre-immunization/vaccination with $C$. dubliniensis (avirulent) or attenuated C. albicans mutants, concomitant with significant reduction in sepsis morbidity. The protection is mediated by innate $\mathrm{Gr}-1^{+}$myeloid-derived suppressor cells that appear to be induced in the bone marrow as a form of trained innate immunity 66,67 . Efforts are underway to extend the understanding of this novel form of innate immune-mediated protection against $C$. albicans bloodstream infections.

In conclusion, the innovative rodent warming/restraining device has been instrumental in advancing our ability to perform i.v. injections of large-scale multi-group animal studies in an efficient and effective manner. As such, we have coined the term, Mouse a Minute, for the device. The device specifications are available from the corresponding author upon request for procurement of a similar device. The techniques demonstrated here could serve as a useful tool in rodent models employing tail vein injections across a broad range of research areas.

\section{Disclosures}

The authors have nothing to disclose.

\section{Acknowledgments}

This work was supported by the LSUHSC Foundation (PLF), and in part by U54 GM104940 from the National Institute of General Medical Sciences of the National Institutes of Health, which funds the Louisiana Clinical and Translational Science Center.

\section{References}

1. Woodard, G. in Methods of animal experimentation Vol. 1 (ed W.J. Gay). 343-359 (1965).

2. Shimizu, S. in The laboratory mouse The handbook of experimental animals (ed Hans J. Hedrich) Ch. 32, 527541 Elsevier Academic Press, (2004).

3. Turner, P. V., Brabb, T., Pekow, C., Vasbinder, M. A. Administration of substances to laboratory animals: routes of administration and factors to consider. Journal of the American Association for Laboratory Animal Science. 50 (5), 600-613 (2011).

4. Turner, P. V., Pekow, C., Vasbinder, M. A., Brabb, T. Administration of substances to laboratory animals: equipment considerations, vehicle selection, and solute preparation. Journal of the American Association for Laboratory Animal Science. 50 (5), 614-627 (2011).

5. Donovan, J., Brown, P. Parenteral injections. Current Protocols in Immunology. Chapter 1, 73 (1) Unit 1, 6, (2006).

6. Schoch, A., Thorey, I. S., Engert, J., Winter, G., Emrich, T. Comparison of the lateral tail vein and the retroorbital venous sinus routes of antibody administration in pharmacokinetic studies. Lab Animal. 43 (3), 95-99 (2014).

7. Jarneborn, A. et al. Tofacitinib treatment aggravates Staphylococcus aureus septic arthritis, but attenuates 
sepsis and enterotoxin induced shock in mice. Scientific Reports. 10 (1), 10891 (2020).

8. Bussey, K. A. et al. Endosomal Toll-like receptors 7 and 9 cooperate in detection of murine Gammaherpesvirus 68 infection. Journal of Virology. 93 (3), (2019).

9. Pitts, M. G., D'Orazio, S. E. F. A Comparison of oral and intravenous mouse models of listeriosis. Pathogens. 7 (1), (2018).

10. Jespersen, $H$. et al. Clinical responses to adoptive Tcell transfer can be modeled in an autologous immunehumanized mouse model. Nature Communications. 8 (1), 707, (2017).

11. Gomez-Cuadrado, L., Tracey, N., Ma, R., Qian, B., Brunton, V. G. Mouse models of metastasis: progress and prospects. Disease Models \& Mechanisms. 10 (9), 1061-1074 (2017).

12. Srinageshwar, B. et al. Surface-modified G4 PAMAM dendrimers cross the blood-brain barrier following multiple tail-vein injections in C57BL/6J mice. ACS Chemical Neuroscience. 10 (9), 4145-4150 (2019).

13. Channabasappa, S. et al. Efficacy of novel antistaphylococcal ectolysin $\mathrm{P} 128$ in a rat model of methicillin-resistant Staphylococcus aureus bacteremia. Antimicrobial Agents and Chemotherapy. 62 (2), (2018).

14. Sadeghi, B. et al. Preclinical toxicity evaluation of clinical grade placenta-derived decidua stromal cells. Frontiers in Immunology. 10, 2685 (2019).

15. Boquet, M. P., Wonganan, P., Dekker, J. D., Croyle, M. A. Influence of method of systemic administration of adenovirus on virus-mediated toxicity: focus on mortality, virus distribution, and drug metabolism. Journal of
Pharmacological and Toxicological Methods. 58 (3), 222-232 (2008).

16. Vines, D. C., Green, D. E., Kudo, G., Keller, H. Evaluation of mouse tail-vein injections both qualitatively and quantitatively on small-animal PET tail scans. Journal of Nuclear Medicine Technology. 39 (4), 264-270 (2011).

17. Lasnon, C., Dugue, A. E., Briand, M., Dutoit, S., Aide, N. Quantifying and correcting for tail vein extravasation in small animal PET scans in cancer research: is there an impact on therapy assessment? EJNMMI Research. 5 (1), 61 (2015).

18. Groman, E. V., Reinhardt, C. P. Method to quantify tail vein injection technique in small animals. Contemporary Topics in Laboratory Animal Science. 43 (1), 35-38 (2004).

19. Aller, M. A. et al. Neuro-immune-endocrine functional system and vascular pathology. Medical Hypotheses. 57 (5), 561-569 (2001)

20. McEwen, B. S. et al. The role of adrenocorticoids as modulators of immune function in health and disease: neural, endocrine and immune interactions. Brain Research. Brain Research Reviews. 23 (1-2), 79-133 (1997).

21. Callewaert, B. L. et al. Absence of arterial phenotype in mice with homozygous slc2A10 missense substitutions. Genesis. 46 (8), 385-389 (2008).

22. Hatakeyama, S., Yamamoto, H., Ohyama, C. Tumor formation assays. Methods in Enzymology. 479, 397-411 (2010).

23. Carlson, R. P. J., Peer B. in In Vivo Models of Inflammation Progress in Inflammation Research eds 
Douglas W Morgan \& Lisa A Marshall) 1-50 Birkhäuser, Basel. (1999).

24. Flecknell, P. in Laboratory Animals: An Introduction for New Experimenters (ed AA Tuffery) 225-260 John Wiley and Sons. (1987).

25. Kim, M. J., Ahituv, N. The hydrodynamic tail vein assay as a tool for the study of liver promoters and enhancers. Methods in Molecular Biology. 1015, 279-289 (2013).

26. Bargellini, A. et al. Effects of chronic exposure to anaesthetic gases on some immune parameters. Science of The Total Environment. 270 (1-3), 149-156 (2001).

27. Elena, G. et al. Inhalatory anesthetic (halothane) associated changes in the immune response in mice. International Journal of Immunopharmacology. 19 (11-12), 699-707 (1998).

28. Buerge, T. in The Laboratory Mouse The handbook of experimental animals (eds H. J. Hedrich, G. Bullock) Ch. 31, 517-526 Elsevier Academic Press. (2004).

29. Cohen, J., Cristofaro, P., Carlet, J., Opal, S. New method of classifying infections in critically ill patients. Critical Care Medicine. 32 (7), 1510-1526 (2004).

30. Wisplinghoff, $\mathrm{H}$. et al. Nosocomial bloodstream infections in US hospitals: analysis of 24,179 cases from a prospective nationwide surveillance study. Clinical Infectious Diseases. 39 (3), 309-317 (2004).

31. Magill, S. S. et al. Multistate point-prevalence survey of health care-associated infections. The New England Journal of Medicine. 370 (13), 1198-1208 (2014).

32. Dupont, H. et al. Predictive factors of mortality due to polymicrobial peritonitis with Candida isolation in peritoneal fluid in critically ill patients. Archives of Surgery. 137 (12), 1341-1346 (2002).
33. Montravers, P. et al. Candida as a risk factor for mortality in peritonitis. Critical Care Medicine. 34 (3), 646-652 (2006).

34. Calandra, T., Bille, J., Schneider, R., Mosimann, F., Francioli, P. Clinical significance of Candida isolated from peritoneum in surgical patients. Lancet. 2 (8677), 1437-1440 (1989).

35. Kojic, E. M., Darouiche, R. O. Candida infections of medical devices. Clinical Microbiology Reviews. 17 (2), 255-267 (2004).

36. Ramage, G., Martinez, J. P., Lopez-Ribot, J. L. Candida biofilms on implanted biomaterials: a clinically significant problem. FEMS Yeast Research. 6 (7), 979-986 (2006).

37. Spellberg, B., Ibrahim, A. S., Edwards, J. E., Jr., Filler, S. G. Mice with disseminated candidiasis die of progressive sepsis. Journal of Infectious Diseases. 192 (2), 336-343 (2005).

38. Conti, H. R., Huppler, A. R., Whibley, N., Gaffen, S. L. Animal models for candidiasis. Current Protocols in Immunology. 105 (1), (2014).

39. Lionakis, M. S., Lim, J. K., Lee, C. C., Murphy, P. M. Organ-specific innate immune responses in a mouse model of invasive candidiasis. Journal of Innate Immunity. 3 (2), 180-199 (2011).

40. Segal, E., Frenkel, M. Experimental in vivo models of candidiasis. Journal of Fungi. 4 (1), (2018).

41. MacCallum, D. M., Odds, F. C. Temporal events in the intravenous challenge model for experimental Candida albicans infections in female mice. Mycoses. 48 (3), 151-161 (2005). 
42. Gordon, C. J. The mouse thermoregulatory system: Its impact on translating biomedical data to humans. Physiology \& Behavior. 179, 55-66 (2017).

43. Mai, S. H. C. et al. Body temperature and mouse scoring systems as surrogate markers of death in cecal ligation and puncture sepsis. Intensive Care Medicine Experimental. 6, 20 (2018).

44. Catty, D., Lehmann, P. F. A simple low-cost restrainer for the intravenous injection of mice. Sabouraudia. 16 (2), 89-90 (1978).

45. Donovan, J., Brown, P. Handling and restraint. Current Protocols in Immunology. Chapter 1 Unit 1, 73 (1), (2006).

46. Dow, H. C. et al. Genetic dissection of intermale aggressive behavior in BALB/cJ and $\mathrm{A} / \mathrm{J}$ mice. Genes Brain and Behavior. 10 (1), (2010).

47. Pugh, P. L., Ahmed, S. F., Smith, M. I., Upton, N., Hunter, A. J. A behavioural characterisation of the FVB/N mouse strain. Behavioural Brain Research. 155 (2), 283-289 (2004).

48. MacCallum, D. M., Odds, F. C. Need for early antifungal treatment confirmed in experimental disseminated Candida albicans infection. Antimicrobial Agents and Chemotherapy. 48 (12), 4911-4914 (2004).

49. Fakhim, H. et al. Comparative virulence of Candida auris with Candida haemulonii, Candida glabrata and Candida albicans in a murine model. Mycoses. 61 (6), 377-382 (2018).

50. Remick, D. G., Newcomb, D. E., Bolgos, G. L., Call, D. R. Comparison of the mortality and inflammatory response of two models of sepsis: lipopolysaccharide vs. cecal ligation and puncture. Shock. 13 (2), 110-116 (2000).
51. Nash, E. E., Peters, B. M., Palmer, G. E., Fidel, P. L., Noverr, M. C. Morphogenesis is not required for Candida albicans-Staphylococcus aureus intraabdominal infection-mediated dissemination and lethal sepsis. Infection and Immunity. 82 (8), 3426-3435, (2014).

52. Rogers, T., Balish, E. Experimental Candida albicans infection in conventional mice and germfree rats. Infection and Immunity. 14 (1), 33-38, (1976).

53. Marquis, G., Montplaisir, S., Pelletier, M., Auger, P., Lapp, W. S. Genetics of resistance to infection with Candida albicans in mice. The British Journal of Experimental Pathology. 69 (5), 651-660 (1988).

54. Ashman, R. B., Fulurija, A., Papadimitriou, J. M. Straindependent differences in host response to Candida albicans infection in mice are related to organ susceptibility and infectious load. Infection and Immunity. 64 (5), 1866-1869 (1996).

55. Ashman, R. B., Bolitho, E. M., Papadimitriou, J. M. Patterns of resistance to Candida albicans in inbred mouse strains. Immunology \& Cell Biology. 71 (3), 221-225 (1993).

56. Liu, Y., Mittal, R., Solis, N. V., Prasadarao, N. V., Filler, S. G. Mechanisms of Candida albicans trafficking to the brain. PLoS Pathogens. 7 (10), e1002305 (2011).

57. Huet, O. et al. Ensuring animal welfare while meeting scientific aims using a murine pneumonia model of septic shock. Shock. 39 (6), 488-494 (2013).

58. Kushimoto, S. et al. The impact of body temperature abnormalities on the disease severity and outcome in patients with severe sepsis: an analysis from a multicenter, prospective survey of severe sepsis. Critical Care. 17 (6), R271 (2013). 
59. Wiewel, M. A. et al. Risk factors, host response and outcome of hypothermic sepsis. Critical Care. 20 (1), 328 (2016).

60. Peters, B. M., Noverr, M. C. Candida albicansStaphylococcus aureus polymicrobial peritonitis modulates host innate immunity. Infection and Immunity. 81 (6), 2178-2189 (2013).

61. Nash, E. E., Peters, B. M., Fidel, P. L., Noverr, M. C. Morphology-Independent Virulence of Candida Species during Polymicrobial Intra-abdominal Infections with Staphylococcus aureus. Infection and Immunity. 84 (1), 90-98 (2016).

62. Hirayama, T. et al. Virulence assessment of six major pathogenic Candida species in the mouse model of invasive candidiasis caused by fungal translocation. Scientific Reports. 10 (1), 3814 (2020).

63. Koh, A. Y., Kohler, J. R., Coggshall, K. T., Van Rooijen, N., Pier, G. B. Mucosal damage and neutropenia are required for Candida albicans dissemination. PLoS Pathogens. 4 (2), e35 (2008).

64. Vergidis, P. et al. Intra-abdominal candidiasis: The importance of early source control and antifungal treatment. PLoS One. 11 (4), e0153247 (2016).

65. Parker, J. C., Jr., McCloskey, J. J., Knauer, K. A. Pathobiologic features of human candidiasis. A common deep mycosis of the brain, heart and kidney in the altered host. American Journal of Clinical Pathology. 65 (6), 991-1000 (1976).

66. Esher, S. K., Fidel, P. L., Jr., Noverr, M. C. Candida/ Staphylococcal Polymicrobial Intra-Abdominal Infection: Pathogenesis and Perspectives for a Novel Form of Trained Innate Immunity. Journal of Fungi. 5 (2), (2019).
67. Lilly, E. A., Ikeh, M., Nash, E. E., Fidel, P. L., Jr., Noverr, M. C. Immune protection against lethal fungal-bacterial intra-abdominal infections. mBio. 9 (1), (2018). 